\title{
The lego way: curve crossing diagrams as general models in physical organic chemistry
}

\author{
Sason S. Shaik \\ Department of Chemistry, Ben-Gurion University, Beer Sheva, 84105, Israel
}

\begin{abstract}
The whole may be understood by its reconstruction from building block components: this is a LEGO principle. A curve crossing diagram is a model for the reconstruction of energy profiles from Valence Bond (VB) building blocks, and may be considered as the quantum chemical analog of Robinson's curved arrow mnemonic. There are two archetypal diagrams which describe chemical transformations: (a) a two-curve diagram in which the spine is the crossing of the two spin-paired covalent forms of the bonds which interchange during the transformation, and (b) a three-curve diagram in which the principal curves (in (a)) are horizontally crossed by an intermediate curve. The two archetypal diagrams constitute a unifying framework for conceptualization and prediction of reactivity patterns, reaction mechanisms and structural trends related to "hypervalency" and "hypercoordination". Examples are discussed.
\end{abstract}

\section{INTRODUCTION}

There is a continuing search in physical organic chemistry for models which can pattern the chemical data and broaden thereby our understanding of chemical phenomena. This quest for understanding has led to the development of two schools of thought which, in the absence of a better terminology, may be called "classical" and "theoretical". Each of these schools has its own particular set of concepts, and experimental methodologies designed to test them.

The classical discipline of physical organic chemistry is a science of rate-equilibrium relationships and had its genesis in the first half of this century, in the works of Brønsted, Hammett, Evans, Polanyi, Bell, and later of Ingold and his school. With some VB ideas and a good deal of healthy empiricism there have emerged important concepts, side by side with their typical experimental methodologies. The Bell-Evans-Polanyi (BEP) principle (ref. 1), the reactivityselectivity principle (ref. 2), the Leffler-Hammond postulate (ref. 3), and the generalized BEMA-HAPOTHLE (ref. 4) are among the concepts which partake in our chemical upbringing and in the shaping of our "chemical intuition".

The theoretical school of physical organic chemistry is based primarily on Molecular Orbital (MO) theory, and had its genesis in the early work of Huckel followed by those of Fukui, Woodward and Hoffmann. By using perturbation theoretic ideas and appealing to the shape and nodal characters of the MO's, important concepts have been formulated and triggered the development of corresponding experimental methodologies. The Huckel-Mobius rules (ref. 5), frontier orbital theory (ref. 6), the principle of orbital symmetry conservation (ref. 7), and so on, are concepts which follow us from our early exposure to chemistry.

The two disciplines coexist, almost as though being two independent sciences. It is not apparent for example, where are the origins of rate-equilibrium relationships or of the Hammond-Leffler postulate in MO theory, nor can the principle of orbital symmetry be traced to anywhere in the underlying conceptual frame of classical physical organic chemistry. Thus, although the two schools are interested in the very same insights, they still seem different like two pieces disjointed from a big jigsaw puzzle. Where does one begin with a search for a new reactivity model? One which not only unifies, and builds bridges between the two disciplines, but can also pattern the data and generate new insights and predictions?

Barriers, transition states and intermediate states are fundamental reactivity features which arise from electronic and nuclear reorganizations. Any model which attempts to conceptualize reactivity must eventually present lucid mechanisms for these reactivity features, and show how the various structure- reactivity patterns spring from these mechanisms. If a mechanism of transition state formation exists one can then analyze the structure and energy of the transition state and relationships which may exist between the transition state and its precursor and successor reactants and products (e.g., rate-equilibrium relationship, and so on). It is apparent then that an effective model must treat simultaneously and on the same footing reactants, products, transition states and intermediate states. 
How do we handle the problem of electronic and nuclear reorganization from a conceptual point of view? In 1979 it has become clear to us that VB theory, although not very popular at that time, is most suited to handle reactivity because of the ability of this theory to treat the fundamental event of a chemical reaction: bond breaking-making. But it was equally clear that VB theory alone is not sufficient, because it is only by use of MO theory that one can show with lucidity the role of local orbital symmetry. The choice fell then upon a VB theory which utilizes for the purpose of spin-pairing both atomic orbitals (AO's) and fragment MO's (ref. 8).

Using this approach we derived in 1981 (ref. 8) the electronic and symmetry principles for the construction of a diagrammatic representation of transition state formation along a reaction coordinate. The main feature of this diagrammatic model is the intended crossing of VB type configurations along the reaction coordinate, in a manner which derives from the nature of the electronic reorganization. In this diagram, the transition state arises from an avoided crossing, or put differently from a resonance mixing of VB configurations which contain reactant-like, product-like as well as other configurations. It was proposed then that avoided crossing is a central mechanism in reactivity projecting the transition state as a unique species which acts, along the reaction coordinate, as the turning point that, by means of resonance mixing, allows the interconversion of the bonding schemes of reactants and products.

The importance of avoided crossing is well known in quantum chemistry (ref. 9), and quite a few models of reactivity have sought criteria to construct avoided crossing diagrams for the purpose of conceptualizing reactivity. For example, Bell, Evans and Polanyi (ref. 1) have used an energy equality criterion to calculate the seam of crossing points of curves which emanate from reactants and products. This has become later the basis of the BEP principle. Marcus (ref. 10) has used disequilibrium and equilibrium solvation structures to model electron transfer reactions with curve crossings. Longuet-Higgins and Abrahamson (ref. 11) and Woodward and Hoffmann (ref. 7) have used an orbital symmetry criterion to reconstruct the avoided crossing for "forbidden" reactions. Salem and collaborators have used imposed symmetry which was then broken in order to construct avoided crossings for photochemical reactivity (ref. 9). Another principle has been used by Devaquet, Sevin and Bigot (ref. 12) who have relied on the "natural correlation" of MO's to illustrate avoided crossings for photochemical reactions. Finally, Warshel has applied curve crossing ideas to biochemical reactions and to reaction dynamics (ref. 13).

Indeed, the interconversion of electron density is central to a chemical transformation, and the insight and generality of a particular model is critically dependent upon the wave functions one uses to model this electron density transformation in terms of avoided crossings. In this respect the present curve crossing model (ref. 8) that uses VB configurations based on a composite of AO's and fragment MO's leads to most general criteria, while at the same time bridges between the concepts of the two schools of physical organic chemistry. This model, later to be called the curve crossing diagram model, has been further developed by the Ben-Gurion group into a general qualitative methodology for the construction of potential energy profiles from their VB type building blocks by principles of quantum mechanical mixing and avoided crossings (ref. 14). This viewpoint has been tested during the years 19821990 and proved useful for understanding reactivity patterns of a variety of organic and organometallic reactions (ref. $14,15)$, and of related problems concerned with the stability of clustered and "hypercoordinated" species relative to their segregated and "normal-coordinated" molecular forms (ref. 14h, 16). The recent development of the multistructure VB program by the Orsay and Polytechnique groups (ref. 17) has allowed to implement the qualitative ideas isomorphically into a rigorous computational scheme (ref. 16a,b; 18). As the applications accumulate they show that a model which is qualitatively useful has also a rigorous and quantitative counterpart.

There are two archetypal diagrams which can be used to conceptualize most chemical transformations. These archetypes are depicted in the curve crossing diagrams in Fig. 1 and 2 which show different patterns of intended crossings along reactions coordinates (RC) which are defined by the geometries of $R$ (reactants) and $P$ (products). Let us postpone the discussion of the various notations of the wave functions and focus on the essential features of the diagrams. Figure 1 shows the intended crossing of two curves which emanate from reactants and products, while Fig. 2 shows the intersection of the same two curves by a third curve of an intermediate character. Avoided crossings in the two archetypes cover the range of chemical phenomena of concerted single step- and stepwise transformations. In the remainder of this lecture we shall discuss the underlying principles for the application of the two diagrams and associate them with specific chemical patterns. For more in depth discussions of the chemical topics we refer to the review articles and the original literature (ref. 14-16).

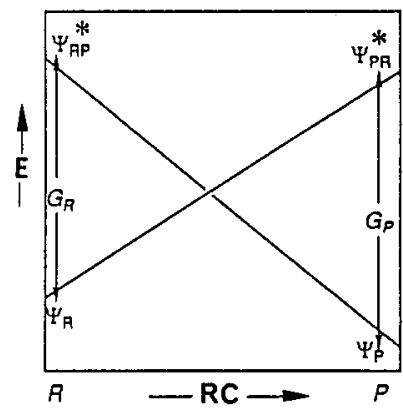

Fig.1. A two-curve diagram

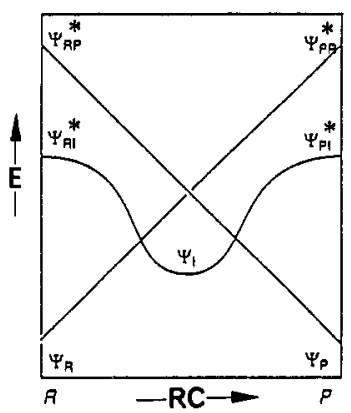

Fig. 2. A three- curve diagram 


\section{THE VALENCE BOND LANGUAGE}

Since we are going to use mostly qualitative ideas based on VB theory it is essential to establish elements of common language by reviewing simple "nuts and bolts" necessary for this lecture. A fuller discussion of VB theory, in the sense used here, has recently appeared (ref. 14d).

\section{Representations of valence bond configurations}

Drawings 1.3 summarize a few representations of two electron bonding. In 1 we show the most common representation of the covalent spin-pairing, also called the Heitler-London (HL) configuration. Here the spin-pairing is represented by the line connecting the two dots. The Lewis structure shown in $\underline{2}$ represents an optimized linear combination of the HL and the ionic structures. In $\mathbf{3}$ we show a representation of a generalized bond-pair which accounts for the singlet spin-pairing of two electrons which reside in two orbitals, which in turn may be AO's in the simplest case and group orbitals in the more complex case. An example of a generalized bond-pair is the singlet pairing between a radical $C^{\cdot}$ and a radical anion $(A-B)^{-}$shown in $\underline{4}$ along with its explicit $A O$ based VB representation in $\mathbf{5}$. It is seen that the bond-pairing is between the singly occupied orbital of $C$ and the $\sigma^{*}$ orbital of the radical anion. From the comparison of $\underline{4}$ and 5 it is seen that the radical anion species $(\mathrm{A}-\mathrm{B})^{-}$is delocalized corresponding to a linear combination of two AO (or atomic hybrid) based VB structures. The generalized bond-pair representation constitutes the key link between the spin-pairing notion of classical VB theory and the orbital symmetry notion of MO theory (ref. $8,14 \mathrm{~d}$ ).

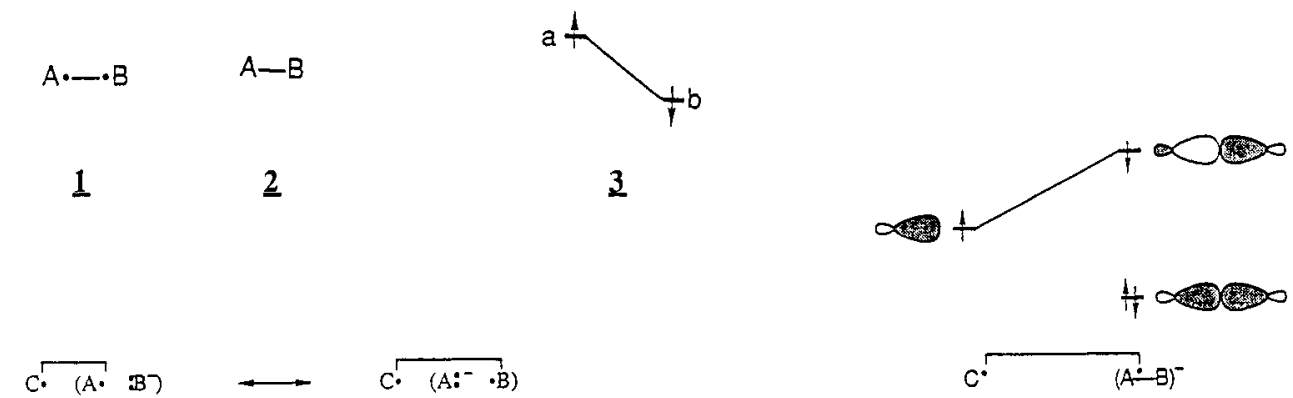

\section{Overlap repulsion in valence bond theory}

Another key element of VB theory is overlap repulsion which is a manifestation of the Pauli exclusion principle, and akin to the three situations depicted in $6-8$. It is seen that the basic "dose" of overlap repulsion for each pair of electrons with identical spins is twice the quantity $h s$, the product of the resonance $(h)$ and overlap $(s)$ integrals of the two overlapping orbitals.

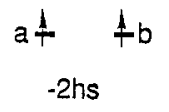

6

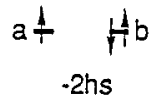

7

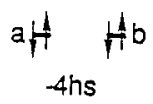

8

\section{Mixing of valence bond structures}

The curve crossing diagrams and the final states after avoided crossing are generated from the mixing of VB structures. The rules for VB mixing are similar to the perturbation theoretic ideas of MO mixing, but the mixing matrix elements in VB theory are more complex. The rules of VB mixing are summarized in ref. 14d, and the most important mixings are: (i) between structures which differ by one electron shift (transfer), and (ii) between structures which differ by a pairwise spin exchange (spin up, spin down $\longrightarrow$ spin down, spin up). In the first case the mixing is proportional to the overlap of the orbitals which participate in the electron shift (transfer), and in the second case to the product of overlaps of the pairs of orbitals which participate in the spin exchange.

\section{TWO CURVE CROSSING DIAGRAMS FOR ONE STEP PROCESSES}

Let us turn to discuss the makeup of the two-curve diagram of Fig. 1. In any single step process the Lewis structure which describes reactants' bonds interchanges with the corresponding structure of the products' bonds. This interchange forms the spine of the two intersecting curves in Fig. 1. There are additional VB structures of secondary nature that mix with each of the curves and contribute to their character (e.g., a long bond character in the case of SN2 reaction, charge transfer configurations in radical attacks, etc.). Thus, the construction of the curve crossing diagram is a LEGO process of piecing up together all the contributing VB structures (ref. 14b,d). We can however waive the detailed construction process by using a simple way of identifying the excited states in the diagram (ref. 8, 14b,d). The excited and ground states are sufficient for the construction of the diagram of Fig.1, because the precise nature of 
the connecting curves (other than having a major Lewis character) should not add at this stage a great deal of vital information.

Each excited state in Fig. 1 is unique in the sense that it involves the same spin-pairing scheme as the ground state at the bottom end of the correlation line. We call these unique excited states the "image states". Thus, each image state can be generated from the ground state directly below it by a particular electronic excitation which prepares for the requisite spin-pairing pattern, while conserving the total spin. The simple principle is that each bond that is broken during the transformation requires one excitation and each bond that is formed requires in turn one corresponding bond-pair. There are two types of elementary excitations which are involved in the image states. The first type is the triplet unpairing (singlet-triplet excitation) of a bond which has to be broken during the transformation, and the second excitation is a vertical charge transfer from the donor center to an empty orbital of the acceptor center.

The choice of the elementary excitation depends on whether or not the reaction centers in question undergo also formal changes in their "oxidation states". The formal oxidation state in our approach is defined with respect to the covalent HL structure. Consider for example, the HL structures for the SN2 reaction in equation 1

$$
\mathrm{Y}^{-}+\mathrm{R} \cdot \mathbf{X} \cdot \mathrm{X} \longrightarrow \mathrm{Y} \cdot-\mathrm{R}+\mathrm{X} \mathbf{:}^{-}
$$

It is seen that Y:- undergoes a formal oxidation - a loss of one electron - while $\cdot \mathrm{X}$ undergoes a formal reduction - a gain of one electron. The reaction is thus defined to involve a formal redox of two reaction centers $(Y, X)$. In this case we utilize a vertical charge transfer excitation from $Y:^{-}$to $R-X$, to generate the pair $Y \cdot /(R-X)^{-}$and we then couple the spins of the two odd electrons as illustrated in $\mathbf{4}$ to obtain a singlet vertical charge transfer state as the image state at the reactant extreme of the diagram in Fig. 1. The exact same procedure is applicable to the product extreme where the image state will be the corresponding charge transfer state $\mathrm{X} * /(\mathrm{R}-\mathrm{Y})^{-}$. In fact, vertical charge transfer states will be the image states for all the reactions or transformations which involve electrophile/nucleophile combinations, and the excitation gaps ( the $G$ 's in Fig. 1) will correspond to vertical charge transfer excitations.

Consider now the "isovalent" transformation which is shown in equation 2 using the HL structures only.

$$
\mathrm{Y}^{\bullet}+\mathrm{R} \cdot \cdot \mathrm{X} \longrightarrow \mathrm{Y}^{\cdot}-\cdot \mathrm{R}+\mathrm{X} \cdot
$$

There is no formal redox in this case, and the image state is obtained therefore by triplet unpairing of the bond and recoupling the electrons of the triplet $R^{\bullet} \uparrow \uparrow^{\bullet} X$ to the odd electron of $Y^{\bullet}$ to generate a bond-pair. The same considerations apply for the reverse process, and here too the image state involves a triplet $\mathrm{RY}$ coupled to $\mathrm{X}^{\circ}$ to form a bond-pair. All radical type reactions and isovalent cycloadditions will behave in a similar manner, using triplet excitations for the image states in the curve crossing diagram.

The following statements summarize the nature of the excitations required to generate the image states in the generalized curve crossing diagram of Fig. 1.

Statement 1: For an isovalent reaction (with bond exchanges only) the image states involve only singlettriplet excitations, one for each bond which has to be broken in the transformation. The odd electrons are coupled into bond-pairs to fit the number of bonds which are formed at the bottom end of the correlation line.

Statement 2: For a reaction which involves a formal redox of reaction centers in addition to bond exchanges, the image states involve one charge transfer excitation for each pair of centers which undergo formal redox. The rest of the excitations will be of the singlet-triplet variety. The odd electrons are coupled into bond-pairs to fit the number of bonds which are formed at the bottom end of the correlation line.

Statement 3: The bond-pairs in the image states must reside in fragment orbitals which are symmetry matched. The symmetry matching of the bond-pairs will influence the nature of the reaction coordinate and transition state structure.

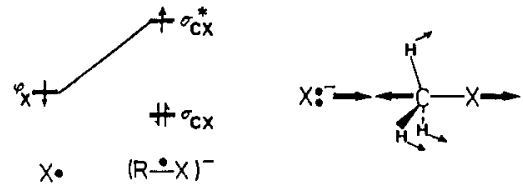

2

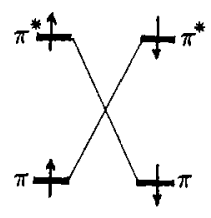

10

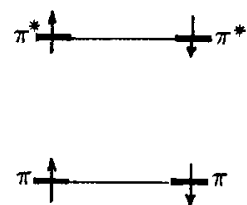

11

Statement 3 can be illustrated by means of two examples. Drawing 2 shows the image state for an identity SN2 reaction. It is seen that the bond-pair resides in the orbital of the nucleophile and in the $\sigma^{*}$ orbital of the R-X molecule. Since a good orbital overlap is required to optimize the bond-pair, this will promote a backside attack of the nucleophile. At the same time, the occupancy of the $\sigma^{*}$ orbital will require the stretching of the R-X bond and the flattening of the valence angles about the $\mathrm{R}$ group in order to relax the radical anion moiety. Taken together, the reaction coordinate will consist of the distortions which are indicated by the heavy arrows in the drawing (ref. 14b,e). 
Another example is the cycloaddition of two ethylene molecules (ref. 14d). In this reaction we need to break two bonds and to make two new ones. Since the reaction is isovalent the image states are constructed by excitation of the two ethylenes to their triplet $\pi \pi^{*}$ states and pairing up the four spins into two bond-pairs. If we pair up the electrons in a $\pi$ MO of one molecule with those in the $\pi^{*}$ MO of the second, as shown in 10 , then in order to symmetry match the fragment orbitals and optimize bonding of the bond-pairs we must proceed along a reaction coordinate which involves a supra-antara mutual orientation of the two ethylene molecules. This is the case of the formally allowed pathway. On the other hand, if we pair up the spins of the electrons in a $\pi-\pi$ and $\pi^{*}-\pi^{*}$ pairwise fashion, as shown in 11 we then require the supra-supra reaction coordinate of the formally forbidden pathway in order to symmetry match the two bond-pairs (note that a mixed type reaction coordinate, a skewed approach which is between supraantara and supra-supra, will enable to enjoy the two types of bond-pairings via a symmetry broken transition state).
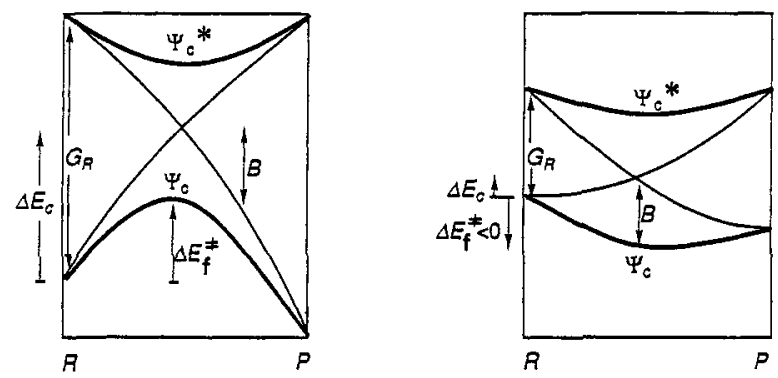

Fig. 3. Avoided crossing diagrams showing how the variation of the excitation gap $\left(\mathrm{G}_{R}\right)$ can lead to transition states $\left(\Delta \mathrm{E}^{\neq}>0\right)$ as well as to stable intermediate states $\left(\Delta \mathrm{E}^{\neq}<0\right)$. These designations refer to $\Psi_{\mathrm{c}}$ on the lower profile.

\section{Reactivity factors: generators of reactivity patterns}

The final reaction profile is obtained by the avoided crossing of the two curves of Fig. 1. Figure 3 shows how the avoided crossing, in the dark lines, generates a pair of states, of which the lowest is stabilized relative to the crossing point by an amount $B$ that is called the avoided crossing interaction. The two avoided crossing diagrams displayed in the Figure illustrate also the impact of the excitation gap. Thus, the general anticipation is that in an isoelectronic series a large gap will result in transition state formation on the lower surface while a very small gap will generate a stable intermediate species on the same surface, with all the tange in between the two extremes.

Isoelectronic series of this kind indeed exist (ref. 14h). For example, the identity transformation of triatomics made of monovalent atoms, $\mathrm{X}^{\cdot}+\mathrm{X}-\mathrm{X}^{\prime} \rightarrow\left[\mathrm{XXX}^{\prime}\right] \rightarrow \mathrm{X}-\mathrm{X}+\mathrm{X}^{\prime}$, exhibits the entire range from $\mathrm{X}_{3}$ transition states to stable intermediate states, as the diagram's excitation gap -- given by the singlet-triplet excitation of the diatomic molecule -- decreases. The qualitative prediction has gained further support from recent ab initio VB computations of the avoided crossing diagram of the two extreme species, $\mathrm{H}_{3}$ and $\mathrm{Li}_{3}$ (the singlet-triplet excitation for $\mathrm{H}-\mathrm{H}$ is 1025 $\mathrm{kJ} / \mathrm{mol}$ while for $\mathrm{Li}-\mathrm{Li}$ it is only $138 \mathrm{~kJ} / \mathrm{mol}$ ) which show that the seemingly counterintuitive prediction of stable intermediate states arising from avoided crossing is well founded (ref. 18b). While such an extreme variation of the gap is not typical in common physical organic series it nevertheless emphasizes the great impact of the excitation gap on chemical reactivity and behavior.

Let us continue in this generalizing spirit and identify the reactivity factors which will in turn be translated into reactivity patterns. A simple equation for the barrier can be deduced from Fig. 3 as a balance between the height of the crossing point $\left(\Delta E_{C}\right)$ and the avoided crossing interaction $(B)$. For the forward direction the barrier becomes equation 3 where in turn the height of the crossing point is expressed as a fraction $f$ of the corresponding diagram gap.

$$
\Delta E_{\mathrm{f}}^{\not \neq}=\Delta E_{c}-B=f G_{R}-B
$$
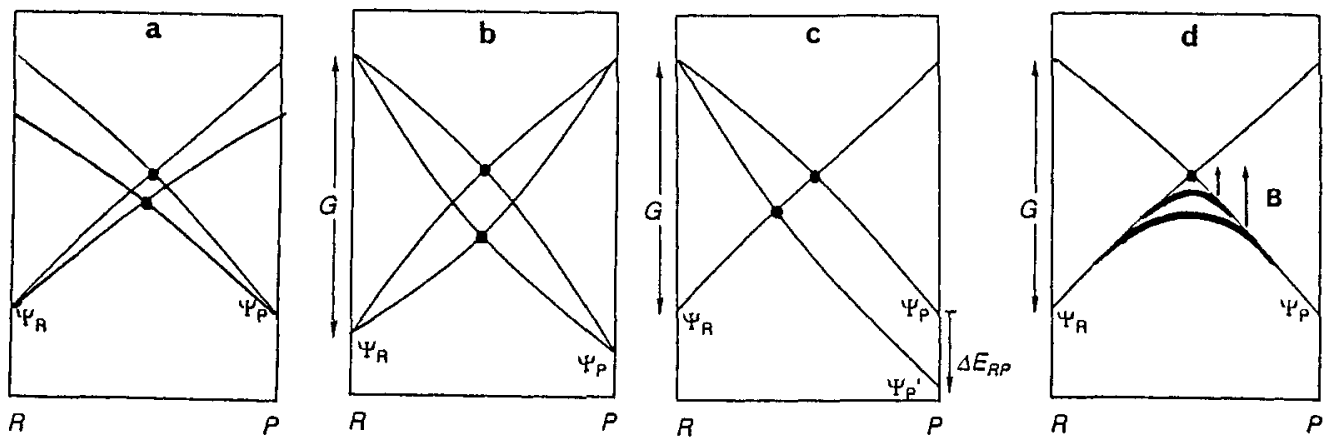

Fig. 4. Curve crossing diagrams showing the effects of: (a) the excitation gaps, (b) the curvature of the curves, (c) the reaction energy (the "thermodynamic driving force"), and (d) the avoided crossing interaction $B$ (the "Quantum Mechanical Resonance Energy"). 
The four curve crossing diagrams in Fig. 4 illustrate the effect of the various reactivity factors in harmony with equation 3. Each part of the Figure schematizes the effect of changing one variable as though all the others do not vary at the same time.

The energy gap effect. As a rule of thumb, the excitation energy gap is related to the strength of the bond(s) that are broken and formed during the transformation. Part (a) of Fig. 4 shows how an increase of the excitation energy gaps leads to a corresponding increase of the crossing point (itself shown by the heavy dot). If all other factors do not vary in an adverse manner then the barrier will increase as the excitation gap increases. This is the "energy gap principle". In most cases the excitation energy gap reflects the gap between the frontier orbitals.

The bond-pair coupling and overlap repulsion effects. Part (b) of Fig. 4 shows that the curvature of the intersecting curves controls the height of the crossing point for a given constant excitation gap, and this determines the size of $f$ in equation 3 because $f$ is simply the ratio of the height of the crossing point to the excitation gap, that is,

$$
f=\Delta E_{c} / G_{R}
$$

The curvature of the curves depends on the steepness of descent of the excited (image) states and on the ascent of the ground states (ref. 14b,d), and occasionally the two factors are linked. Quite a few classical as well as quantum chemical interactions affect the curvature (e.g., the makeup of the VB configurations of the curves, steric effects , electrostatic effects, etc.), and we discuss here two quantum chemical effects which appear to be of key importance.

The steepness of descent of the image states appears to be dominated by the strength of the coupling of the bondpairs which must become localized bonds at the bottom end of each curve. When the bond-pair electrons reside in delocalized fragment orbitals (see for example drawings 4 and 2 ) the bond-pair coupling, along the reaction coordinate, is inhibited and the curves will descend in a shallow manner leading to a high crossing point for a given constant gap, that is a large $f$ factor in equation 3. If this is the dominant variation in a series then the barrier will increase as the orbitals in which the bond-pairs reside, in the image state, become more delocalized. This is the "bond-pair coupling principle".

The steepness of ascent of the curves from their ground states depends on the overlap repulsion caused by electrons with identical spins (see 6-8), while the descent depends, as just discussed, on the singlet coupling of bond-pairs. Atoms which are strong binders have a high ratio, $\alpha$, of overlap repulsion to singlet-pairing attraction, while very weak binders have a small $\alpha$. As we have recently shown (ref. 18b), the $f$ factor in isovalent reactions is proportional to $\alpha$, and reactions or transformations of weak binders will involve generally smaller $f$ factors in equation 3 . This is an important principle (yet unnamed) that outlines the switch of reactivity patterns from transition state species for strong binders to stable clusters for weak binders (ref. 14h).

The rate-equilibrium effects. Part (c) of Fig. 4 illustrates the lowering of the crossing point, i.e., decreasing $f$ in equations 3 and 4 , by making the reaction energy more negative. It is seen that in the absence of an adverse variation of the other reactivity factors, then making the reaction more exothermic will lower also its barrier. This is the well known BEP principle which forms one of the foundations of classical physical organic chemistry.

The quantum mechanical resonance energy (QMRE) effect. Part (d) of Fig. 4 depicts the effect of the degree of avoided crossing $(B)$ on the height of the barrier; the larger the $B$ the smaller the barrier. The avoided crossing interaction is due to mixing of two bonding schemes at the crossing point and hence we refer to $B$ also as the QMRE of the transition state.

Using classical VB theory it is possible to show that (ref. 19) the QMRE is proportional to the strength of the various bonds in the transition state. The QMRE can also be related to the energy gap between the two states which result from the avoided crossing (ref. 14d;19). This gap can be translated in turn to the corresponding MO gap between the HOMO and LUMO of the transition state itself, as shown in equation 5, where the $S$ refers to the overlap of the two bonding schemes at the crossing point.

$$
B=\left[\left(1-S_{12}\right) / 2\right]\left(\mathrm{E}_{\mathrm{LUMO}^{-}} \mathrm{E}_{\mathrm{HOMO}}\right)
$$

This latter expression illuminates the connection between the avoided crossing diagrams and the orbital symmetry rules (ref. 7). Thus, since formally "allowed" transition states possess much larger HOMO-LUMO gaps than their formally "forbidden" analogs, the equation predicts that $B$ ("allowed") is larger than $B$ ("forbidden"). This highlights also the notion of "aromaticity" and "antiaromaticity" of transition states (ref. 20). Computations of QMRE's reveal that indeed this property is larger for aromatic situations relative to antiaromatic situations (ref. 14h). The general effect of the magnitude of $B$ on reactivity can be termed "the QMRE principle".

Equation 6 is an expression which shows the explicit dependence of the barrier on all the reactivity factors; the two different diagram gaps, two independent $f$ factors and a nonzero reaction energy/thermodynamics $\left(\triangle E_{R P}\right)$.

$$
\Delta E^{\neq}=\left[\left(f_{R}^{\prime}+f_{P}^{\prime}\right)\left(G_{P}+\Delta E_{R P}\right) /\left(G_{R}+G_{P}\right)\right] G_{R}-B
$$


The $f^{\prime}$ factors are curvature factors of the curves which emanate from the reactants and from the products with corrections due to the nonzero reaction thermodynamics (ref. $14 \mathrm{~d}, \mathrm{~g}$ ). This explicit equation is related to equation 3 , and it may be seen that the $f$ in equation 3 is an effective parameter which takes into account the curvatures of the two curves as well as the asymmetry of the two diagram gaps. In a case where the diagram gaps are equal and the reaction energy is zero, the $f$ in equation 3 is simply the algebraic average of the two curvature factors in equation 6 . Equations 4 and 6 are connected also to the Marcus equation (ref. 10). For example, using in equation 6 two parabolas with $f^{\prime}$ $=1 / 4$ and assuming two identical curves with a zero avoided crossing, leads to the Marcus equation for an electron transfer process (in terms of the optical charge transfer transition). The avoided crossing model is thus seen to build bridges between different theoretical models and to offer a unified outlook of reactivity.

\section{Reactivity patterns}

The foregoing reactivity factors have all clear chemical identities and will generate in turn corresponding reactivity patterns. Reactivity as a whole can be conceptualized and predicted as a manifestation of the intricate interplay of the reactivity factors.

Manifestations of "the energy gap principle". Reaction series which follow the energy gap principle are abundant in the seminal work of Kochi on charge transfer activation (ref. 21). Patterns which obey the energy gap principle have been observed by us in SN2 reactivity (ref. $14 \mathrm{~b}, \mathrm{e}, \mathrm{g}$ ), in nucleophilic vinylic attacks (ref. 15a), in electrophilenucleophile reactivities (ref. 15b,c) and in models of radical exchange reactions (ref. 14h). In "reaction families", i.e., series which obey the "energy gap principle" due to the constancy of the $f$ and $B$ quantities in equation 3 (ref. 14d, $15 \mathrm{~b}, \mathrm{c} ; 19)$, it is possible to determine an experimental value for the QMRE of the transition states in the family. This quantity may eventually be translated to a corresponding transition state geometry (ref. 19).

Interplay between reactivity factors. Oftentimes all the reactivity factors change simultaneously in a group of reactions, and reactivity will become an intricate interplay of patterns, as illustrated with examples from SN2 reactivity and radical addition to olefins.

The identity SN2 reaction, equation 7 , should initially be discussed in terms of the quantities $f, G$, and $B$ in equation 3 (ref. $14 \mathrm{~b}, \mathrm{e}, \mathrm{g} ; 22$ ).

$$
\mathrm{X}:^{-}+\mathrm{CH}_{3} \mathrm{X}^{\prime} \longrightarrow \mathrm{XCH}_{3}+: \mathrm{X}^{\prime-}
$$

Since the $\mathrm{C}-\mathrm{X}$ bond energies do not vary in a wide range, and since these bonds are stretched in the transition state (it turns out actually that the stronger the bond the greater its percentage of stretching) it was reasoned that the avoided crossing interaction $B$ would be a "passive" variable, one that does not affect relative reactivity in the series. This leaves the vertical charge transfer excitation gap and the $f$ factor as the organizing quantities of reactivity.

In the gas phase, the charge transfer energy gap -- given by the difference between the vertical ionization potential, $I^{*}$, of the anion and the vertical electron affinity, $A^{*}$, of the molecule (see the charge transfer state in 2 ) - is proportional to the bond strength $(D)$ in the molecule, and is very roughly given by equation 8 .

$$
I_{\mathrm{X}:}{ }^{*}-A_{\mathrm{MeX}}{ }^{*} \sim(3 / 2) D_{\mathrm{Me}-\mathrm{X}}
$$

What about the $f$ factor? Our original postulate in 1982 (ref. 22) was that this factor depends on the bond-pair coupling effect. The bond-pair in the charge transfer state in 9 is delocalized in the sense that is shown above in 4 and 5. Drawing 12 illustrates the effect of this electron delocalization by inspecting the bond-pair interaction between the radical $\mathrm{X}^{\cdot}$ and the radical anion $(\mathrm{R}-\mathrm{X})^{-}$. It is seen that electron delocalization in the radical anion inhibits the bondpair coupling because one of the component VB configurations possesses two electrons on the $\mathrm{R}$ union center, and this imposes repulsive three electron interaction in proportion to the weight of the configuration $(\mathrm{R}:-\cdot \mathrm{X})$ which causes the repulsion. The larger this weight the weaker the bond-pair coupling and the larger the $f$ factor. In general then, the $f$ factor in SN2 follows the bond-pair coupling principle.

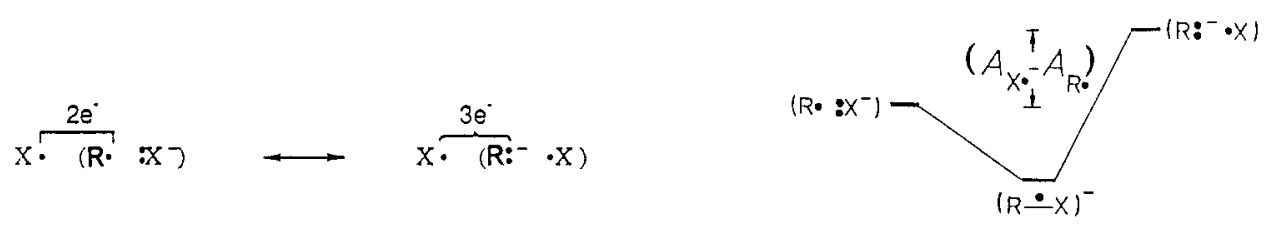

The weight, $W_{\mathrm{R}}$ of the repulsive configuration, $(\mathrm{R}: \cdots \mathrm{X})$ in the radical anion is inversely proportional to the energy difference between the configurations in the VB mixing. In the configuration mixing diagram in 13 this factor is given by the difference between the group electron affinities of $R \cdot$ and $\cdot X$. For a fixed $R$ group, being methyl, the $W_{R}$ : will be inversely proportional to the electron affinity of the $X$ group, that is,

$$
W_{\mathrm{R}:} \propto 1 / A_{\mathrm{X}}
$$


Reactivity in the identity SN2 reaction can therefore be conceptualized in terms of the variation of the two thermochemical quantities (equations 8 and 9 ) i.e., the $C-X$ bond energy and the $\cdot X$ group electron affinity. The stronger the $C-X$ bond and the smaller the $\cdot X$ group electron affinity the higher the barrier (ref. 14e). Thus, identity SN2 barriers will generally decrease as the group $X$ changes down a column and from left to right in a row in the periodic table.

Many more aspects of SN2 reaction and its transition state are discussed with excruciating details in the various reviews and a forthcoming monograph (ref. $14 \mathrm{~b}, \mathrm{e}, \mathrm{g}$ ). These aspects include the geometry of the transition state, the following and breakdowns of the BEP principle, the effect of solvent, nucleophilicity and leaving group ability, and reactivity as well as reactivity-selectivity crossovers. All these aspects, and some new fundamental relationships between transition state geometries and barriers (ref. 23), can be understood in terms of an activation mechanism which consists of molecular distortions and solvent reorganization which are required to promote the resonance between the ground and charge transfer states; a mechanism which in short is called "single electron shift" . The transition state in a single electron shift mechanism possesses a large QMRE in contrast with the single electron transfer kin which possesses a QMRE which is extremely small, $<4 \mathrm{~kJ} / \mathrm{mol}$ (ref. 14a-c, e,f).

The interplay of reactivity factors shapes also the regioselectivity patterns in radical addition to olefins. The major regiochemical pathway follows the "bond-pair coupling principle" (ref. 15d). Thus, the preferred regiochemical pathway is the olefinic site with the highest triplet spin density which thereby optimizes the bond-pair interaction. This site happens to be the less substituted site, as depicted in 14 which shows that the substituted site carries two electrons in the contributing zwitterionic VB structure of the triplet $\pi \pi^{*}$ olefin. This two electron occupancy on the substituted site contributes a repulsive three electron interaction in the bond-pair with the attacking radical, and this in turn means a larger barrier for the attack on this site owing to larger $f$ factors in equation 6 . Regioselectivity zigzags and inverted regioselectivities occur whenever the BEP factor (reaction thermodynamics) has a strong and opposite directive effect compared with the bond-pair coupling factor. Such regiochemical inversions and zigzags occur in the reactions of trifluoroethylene with $\mathrm{CF}_{3}, \mathrm{CH}_{3}, \mathrm{H}$ and so on (ref. 15d).

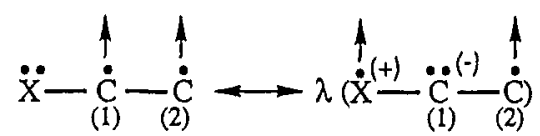

14

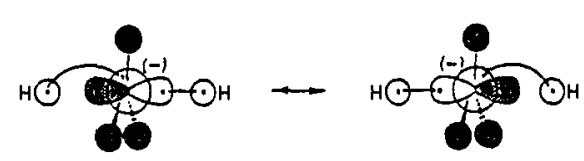

15

Manifestations of "the QMRE principle". Stereochemistry is really in the heart of chemistry, and it is here where MO theory has a traditional edge over VB theory. However, since our VB approach uses a blend of AO's and fragment MO's we can easily recognize that the role of orbital symmetry is embedded in the avoided crossing interaction, the QMRE of the transition state, as discussed above (equation 5).

Since the avoided crossing diagram is based in essence on the VB mixing motif, this allows to make stereochemical predictions also for odd electron reactions where MO theory will not give a clear cut answer. Consider for example, the nucleophilic cleavage of one electron bonds in equation 10.

$$
\mathrm{Nu}:+\mathrm{RR}^{\prime} \mathrm{R}^{\prime \prime} \mathrm{X}^{+} \longrightarrow{ }^{+} \mathrm{Nu}-\mathrm{CRR} \mathrm{R}^{\prime \prime}+\cdot \mathrm{X}
$$

Following Statement 1 , the image state in the avoided crossing diagram involves the triplet excited state of the C-X two-electron bond, coupled to the radical form of the nucleophile to a total of doublet spin (ref. 15f). The avoided crossing interaction arises due to the resonance mixing of the main two configurations at the crossing point: the ground state ( $\mathrm{Nu}$ : and the $\mathrm{RR}^{\prime} \mathrm{R}^{\prime \prime} \mathrm{C}^{\cdot} \mathrm{X}^{+}$radical cation) and the image state. Following the VB mixing rules, briefly mentioned above, the mixing between the configurations and hence also the QMRE will be proportional to the overlap of the nucleophilic orbital with the $\sigma^{*}$ orbital of the radical cation moiety i.e.; the two orbitals which participate in the single electron shift between the two configurations. The $\sigma^{*} \mathrm{MO}$ is predicted then to control the stereochemical pathway of the nucleophilic cleavage reaction leading to a backside attack with inversion of configuration at the site of attack (ref. 24). The only known experimental results as yet, by Dinnocenzo et al (ref. 25), support the prediction.

\section{THREE CURVE CROSSING DIAGRAMS FOR STEPWISE PROCESSES}

The component steps in a stepwise transformation can be discussed by use of a two-curve model (Fig. 1) for each of the steps. A more complete understanding is achieved however by analyzing the problem with the aid of the threecurve model in Fig. 2 which assembles the steps in a single diagram and exhibits a double curve crossing.

In Fig. 2 the transformation from $R$ to $P$ is mediated by a third curve which crosses the principal curves that emanate from $R$ and $P$. This third curve is anchored at two excited states which possess characters which are different than the classical normal coordinated Lewis structures of $R$ and $P$. There are many such excited states, and they mix into 
the principal curves of the single step process to endow the transition state with their characters (ref. 14a-c). Occasionally, however, one of these nonunique excited states is stabilized and descends low enough to cross the two principal curves, significantly below their own crossing point. This new crossing structure defines an intermediate species which mediates the stepwise transformation from $R$ to $P$. This is the basis for the transition from a concerted single step to a stepwise mechanism (e.g., SN2 $\rightarrow$ SN1) in physical organic chemistry, and as it has turned out this is also the basis for description of the phenomenon of hypercoordination / hypervalency.

\section{Types of intermediate states}

In our work we have encountered intermediate states of two types.

Intermediates due to covalent-ionic avoided crossings. A prototype of this avoided crossing is the simple transformation $\mathrm{F}^{-}+\mathrm{H}-\mathrm{F}^{\prime} \rightarrow \mathrm{F}-\mathrm{H}+\mathrm{F}^{\prime}$, which involves a stable (FHF') ${ }^{-}$intermediate. Recent VB computations by the Orsay group show that this intermediate arises from the crossing of the two covalent HL structures (defining the principal curves) by the triple-ionic form $\mathrm{F}^{-} \mathrm{H}^{+} \mathrm{F}^{\prime-}$ (defining the horizontal intermediate curve). The stability of the triple-ion structure at the clustered geometry arises mainly due to the small "size" of the $\mathrm{H}^{+}$which therefore allows a very close approach of the $\mathrm{F}^{-}$ions and a huge electrostatic stabilization. The same type of crossing is the root cause for the SN1, and E1 mechanisms, only that in these cases the positive ion is a much more encumbered carbenium ion which does not allow a compact triple-ion structure with significant electrostatic stabilization. Here the solvent comes to the fore and by stabilizing the triple ion leads to its crossing below the crossing point of the two covalent forms. A similar description applies for the E1cB mechanism.

Intermediates due to avoided crossings with "relay-states". At times the concerted mechanism requires too high a barrier, and an intermediate relay-state -- one which utilizes relay groups or substituents to reorganize the electrons -intervenes and crosses the principal curves to mediate the process with a lower barrier. The relay groups in the intermediate states remain net intact in the overall transformation, but they actually participate in the electronic reorganization. An example for such a stepwise mechanism is the SNV (nucleophilic substitution on a vinylic system, $\mathrm{Nu}:+\mathrm{RR} \mathrm{R}^{\prime} \mathrm{C}=\mathrm{CR}^{\prime \prime} \mathrm{X} \rightarrow \mathrm{RR} \mathrm{R}^{\prime} \mathrm{C}=\mathrm{CR} \mathrm{R}^{\prime \prime} \mathrm{Nu}+\mathrm{X}:$ ). Here the direct substitution involves principal curves anchored at the $\sigma$ charge transfer states in analogy with the SN2 mechanism (see 2). The intermediate curve is anchored at the $\pi$ charge transfer state (ref. 15a), and it is this state that gives rise to the intermediacy of carbanions in the substitution process (ref. 26). The SRN1 mechanism is of a similar origin which by disruption of bond-pair coupling results in a radical plus radical anion species, in an intermediate step of the mechanism (ref. 14b).

Hypercoordination / hypervalency as an avoided crossing phenomenon. We have recently started in collaboration with the Orsay group to explore the root causes of "hypervalency" (ref. 16). What emerges at this point is that the major mechanism of "hypervalency" involves a horizontal crossing of the normal-valent Lewis structures, by a third structure which originates from a valence excited state, devoid of d-AO's, that is stabilized in the hypercoordinated geometry significantly below the crossing point of the Lewis or purely covalent curves. This is the root cause for the stability of the hypercoordinated, and formally nine electron, $\mathrm{PH}_{4}, \mathrm{AsCl}_{4}$, etc., radicals (ref. $16 \mathrm{c}$ ), and the formally 10 electron anions of pentacoordinated $\mathrm{Si}(\mathrm{ref} .16 \mathrm{a}, \mathrm{b})$.

An example along these lines is the comparison of the $\mathrm{SiH}_{5}$ radical and anion species. The former is a transition state (ref. 27) for the $\mathrm{H}$ exchange reaction, while the latter is a stable species about $80 \mathrm{~kJ} / \mathrm{mol}$ more stable than $\mathrm{H}^{-}+\mathrm{SiH}_{4}$ (ref. 28). This comparison is interesting and reminiscent of the Huckel rules in the sense that stability is electron count dependent. A VB computation of the curve crossing diagram (ref. 16a,b) shows that $\mathrm{SiH}_{5}{ }^{-}$owes its stability to the VB structure depicted above in $\mathbf{1 5}$ which crosses horizontally much below the crossing point of the normal-valent Lewis curves (describing $\mathrm{H}:^{-}+\mathrm{SiH}_{4}$ ). The structure 15 possesses two resonating axial bonds; one formed with the $\mathrm{p}$ $\mathrm{AO}$ of $\mathrm{Si}$ and the other with the $\sigma^{*}$ orbital of the equatorial $\mathrm{SiH}_{3}$ moiety. Thus, the 10 th valence electron finds comfortable occupancy in the $\sigma^{*}$ orbital of the equatorial $\mathrm{SiH}_{3}$ moiety and can form thereby an extra axial bond. Going to the corresponding $\mathrm{SiH}_{5}$ radical, this $10 \mathrm{th}$ electron is removed and the hypercoordinated VB structure, $\mathbf{1 5}$, disappears. In its absence $\mathrm{SiH}_{5}$ becomes a normal transition state due to the avoided crossing of the normal coordinated Lewis curves.

\section{CONCLUDING REMARKS}

Barriers, transition state structures, structure-reactitvity relationships, reaction mechanisms, and hypercoordination...This collage of chemical problems which are presented in this lecture gives a flavor of the range of applicability of the curve crossing model in terms of just the two archetypal diagrams in Fig. 1 and 2. Indeed, the model forms a unified basis for conceptualization of chemical phenomena which involve species connected via reaction coordinates, and related by electronic and bond reorganizations. This in essence is what physical organic chemistry is concerned with.

The merits of the model derive from two main features. Firstly, the curve crossing diagrams are expressions of the electronic reorganization which attends the chemical transformation, and in this sense these diagrams are the quantum chemical analogs of Robinson's curved arrow mnemonic with the energetic and structural dimensions added. Secondly, the construction of the diagram is a LEGO process based on quantum mechanical mixing rules and on the same type of philosophy that had made MO theory so successful (ref. 29): that the whole may be understood by its reconstruction from its building block components.

With the new capabilities, to test the qualitative model in a quantitative and an isomorphic manner and to unravel new features to which the qualitative model is naturally blind, we are looking forward to the forthcoming insight. 


\section{Acknowledgement}

The research was supported in the years 1989-1990 by the Basic Research Foundation Administered by the Israel Academy of Sciences and Humanities. I am grateful for lasting and exciting associations with A. Pross and P. C. Hiberty and am indebted to all my other collaborators whose contributions made this lecture possible; R. Bar, A. Bartuv, E. Buncel, E. Canadell, D. Cohen, A. Demoliens, J. P. Dinnocenzo, E. Duzy, L. Eberson, O. Eisenstein, J. M. Lefour, P. Maitre, G. Ohanessian, H. B. Schlegel, G. Sini, I. H. Um, F. Volatron and S. Wolfe.

\section{REFERENCES}

1. (a) M.G. Evans and M. Polanyi, Trans. Far. Soc. 34, 11 (1938). (b) R. P. Bell, Proc. Roy. Soc. London A 154. 414 (1936).

2. A. Pross, Adv. Phys. Org. Chem, 14, 69 (1977).

3. (a) G. S. Hammond, J. Am. Chem. Soc. 77, 334 (1955). (b) J. E. Leffler, Science (Washington. D. C.) 117,340 (1953).

4. W. P. Jencks, Chem. Rev. 85, 511 (1985).

5. (a) J. P. Garratt, Aromaticity, Wiley, New York (1986). (b) E. Heilbronner, Tetrahedron Lett. 1923 (1964).

6. K. Fukui, Acc. Chem. Res. 4. 57 (1971).

7. R. B. Woodward and R. Hoffmann, The Conservation of Orbital Symmetry, Academic Press, New York (1970).

8. S. S. Shaik, J. Am. Chem. Soc. 103, 3692 (1981).

9. (a) L. Salem, C. Leforestier, G. Segal and R. Wetmore, J. Am. Chem. Soc. 97,479 (1975). (b) L. Salem, Science (Washington, D. C.) 191,822 (1976). (c) W. G. Dauben, L. Salem and N.J. Turro, Acc. Chem. Res. 8, 41 (1975).

10. R. A. Marcus, Annu. Rev. Phys. Chem. 15.155 (1964).

11. H. C. Longuet-Higgins and E. W. Abrahamson, J. Am. Chem. Soc. 87, 2045 (1965).

12. A. Devaquet, A. Sevin and B. Bigot, J. Am. Chem. Soc. 100, 2009 (1978).

13. A. Warshel, Acc. Chem. Res. 14.284(1981).

14. (a) A. Pross and S. S. Shaik, Acc. Chem. Res. 16. 363 (1983). (b) S. S. Shaik, Prog. Phys. Org. Chem. 15 , 197 (1985). (c) A. Pross, Adv. Phys. Org. Chem. 21, 99 (1985). (d) S. S. Shaik, in: New Concepts for Understanding Organic Reactions, J. Bertran and G. I. Csizmadia, Eds., ASI NATO Series, C267, Kluwer, Dordrecht, 1989. (e) S. S. Shaik, Acta Chem. Scand. 44, 205 (1990). (f) A. Pross, Acc. Chem. Res. 18, 212 (1985). (g) S. S. Shaik, H. B. Schlegel and S. Wolfe, Theoretical Aspects of Physical Organic Chemistry. The SN2 Transition State. Wiley, in press (1991). (h) S. S. Shaik, P. C. Hiberty, G. Ohanessian and J. M. Lefour, J. Phys. Chem. 92. 5086 (1988). (i) S. S. Shaik and P. C. Hiberty, in: Theoretical Models for Chemical Bonding, Vol. 4. Z. B. Maksic, Ed., Springer Verlag, Heidelberg (1991).

15. (a) D. Cohen, R. Bar and S. S. Shaik, J. Am. Chem. Soc. 108, 231 (1986). (b) S. S. Shaik, J. Org. Chem. 52. 1563 (1987). (c) E. Buncel, S. S. Shaik, I.H. Um and S. Wolfe, J. Am. Chem. Soc. 110,1275 (1988). (d) S. S. Shaik and E. Canadell, J. Am. Chem. Soc. 112, 1446 (1990). (e) L. Eberson and S.S. Shaik, J. Am. Chem. Soc. 112, 4484 (1990). (f) S. S. Shaik and A. Pross, J. Am. Chem. Soc, 111, 4306 (1989).(g) A. J. Shusterman, I. Tamir and A. Pross, J. Organomet. Chem. 340.203 (1988).

16. (a) G. Sini, P.C. Hiberty and S. S. Shaik, J.Chem. Soc. Chem. Commun. 772 (1989). (b) G. Sini, G. Ohanessian, P.C. Hiberty, and S. S. Shaik, J.Am. Chem. Soc. 112. 1407 (1990). (c) A. Demoliens, O. Eisenstein, P.C. Hiberty, J.M. Lefour, G. Ohanessian, S. S. Shaik and F. Volatron, J. Am. Chem. Soc. 111. $5623(1989)$.

17. P.C. Hiberty and J. M. Lefour, J. Chim. Phys. 84, 607 (1987).

18. (a) G. Sini, S. S. Shaik, J.M. Lefour, G. Ohanessian and P.C. Hiberty, J. Phys. Chem. 93, 5661 (1989).

(b) P. Maitre, P.C. Hiberty, G. Ohanessian and S. S. Shaik, J. Phys. Chem. 94.4089 (1990).

19. S. S. Shaik, E. Duzy and A. Bartuv, J. Phys. Chem. 94, in press (1990).

20. (a) M. J. S. Dewar, Angew. Chem. Int. Ed. Engl. 10, 761 (1971). (b) H.E. Zimmerman, J.Am.Chem.Soc. 88, $1564,1566(1966)$.

21. J. K. Kochi, Angew. Chem. Int. Ed. Engl. 27, 1227 (1988).

22. (a) S. S. Shaik, New J. Chem. 6,159 (1982). (b) S. S. Shaik and A. Pross, J. Am. Chem. Soc. 104, 2708 (1982).

23. S. S. Shaik, H. B. Schlegel and S. Wolfe, J. Chem. Soc. Chem. Commun. 1322 (1988).

24. S. S. Shaik and J. P. Dinnocenzo, J. Org. Chem. 55, 3434 (1990).

25. J. P. Dinnocenzo, W. P. Todd, T. R. Simpson and I. R. Gould, J.Am.Chem.Soc. 112. 2462 (1990).

26. Z. Rappoport, Acc. Chem. Res. 14. 7 (1981).

27. F. Volatron, P. Maitre and M. Pellisier, Chem. Phys. Lett. 166, 49 (1990).

28. D. J. Hajdasz and R. R. Squires, J. Am. Chem. Soc. 108,3139 (1986).

29. R. Hoffmann, Science (Washington. D. C.) 211, 995 (1981). 